\title{
Online Social Networking, Sexual Risk and Protective Behaviors: Considerations for Clinicians and Researchers
}

\author{
Ian W. Holloway • Shannon Dunlap • \\ Homero E. del Pino • Keith Hermanstyne • \\ Craig Pulsipher • Raphael J. Landovitz
}

Published online: 19 June 2014

(C) Springer International Publishing AG 2014

\begin{abstract}
Online social networking refers to the use of internet-based technologies that facilitate connection and communication between users. These platforms may be accessed via computer or mobile device (e.g., tablet, smartphone); communication between users may include linking of profiles, posting of text, photo and video content, instant messaging, and email. This review provides an overview of recent research on the relationship between online social networking and sexual risk and protective behaviors, with a focus on use of social networking sites (SNS) among young people and populations at high risk for sexually
\end{abstract}

\section{W. Holloway $(\triangle)$}

Department of Social Welfare, UCLA Luskin School of Public

Affairs, 3250 Charles Young Drive, Los Angeles, CA 90095, USA

e-mail: holloway@luskin.ucla.edu

S. Dunlap $\cdot$ C. Pulsipher

UCLA Luskin School of Public Affairs, Los Angeles, CA, USA

S. Dunlap

e-mail: shandunlap@ucla.edu

C. Pulsipher

e-mail: cpulsi@ucla.edu

H. E. del Pino

Psychiatry and Human Behaviors, Charles R. Drew University of Medicine and Science, 1731 E. 120th St., Building N, Los Angeles, CA 90059, USA

e-mail: homerodelpino@cdrewu.edu

\section{K. Hermanstyne}

Robert Wood Johnson Foundation Clinical Scholars Program, University of California, Los Angeles, 10920 Wilshire Boulevard, Suite 710, Los Angeles, CA 90024, USA

e-mail: KHermanstyne@mednet.ucla.edu

\section{R. J. Landovitz}

Division of Infectious Diseases, David Geffen School of Medicine, 10833 Le Conte Ave, Los Angeles, CA 90095, USA

e-mail: RLandovitz@mednet.ucla.edu transmitted infections (STIs). While findings are mixed, the widespread use of SNS for sexual communication and partner seeking presents opportunities for the delivery and evaluation of public health interventions. Results of SNS-based interventions to reduce sexual risk are synthesized in order to offer hands-on advice for clinicians and researchers interested in engaging patients and study participants via online social networking.

Keywords Internet · Online $\cdot$ Social networking $\cdot$ SNS · Social media $\cdot$ New media $\cdot$ Sexual risk $\cdot$ STI $\cdot$ HIV . Facebook · MySpace · Twitter · Grindr · Youth · MSM . Smartphone $\cdot$ Tablet $\cdot$ Geosocial networking applications . Apps $\cdot$ Technology

\section{Introduction}

Online social networking refers to the use of internet-based technologies that facilitate connection and communication between users. These platforms may be accessed via computer or mobile device (e.g., tablet, smartphone); communication between users may include linking of online profiles, posting of text, photo and video content, instant messaging, and email. The popularity of online social networking sites (SNS) has grown rapidly in recent years. For instance, SNS use by adults 18 years of age and older has risen dramatically from $8 \%$ in 2005 to $72 \%$ in 2013 [1]. Recent data suggests that $23-68 \%$ of people in developing countries use the internet and among these users, a median of $77 \%$ use SNS [2]. SNS, such as Facebook, Twitter, Instagram, Pinterest, and many others, have become embedded in American culture. Nearly threefourths of all internet users in the USA, regardless of age, gender, or ethnicity, use at least one SNS, and over $40 \%$ use two or more SNS [3]. While these data indicate the growing popularity of SNS across the population as a whole, young 
people between the ages of 18 to 29 are the heaviest SNS users [4]. SNS use rose sharply between 2005 and 2013 for young adults (ages 18-29), whose use increased from $9 \%$ to $89 \%$ [1], and for teens (ages 12-17), who experienced a more modest increase from $55 \%$ in 2006 to $81 \%$ in 2012 [5].

SNS are used for a variety of reasons, including maintenance of pre-existing social ties and formation of new ties based on shared interests. Clinicians and health researchers have paid close attention to the ways in which SNS may facilitate sexual partnering and influence sexual risk behaviors. The literature has focused primarily on youth, as well as gay, bisexual and other men who have sex with men (hereafter MSM), two groups at disproportionate risk for acquiring sexually transmitted infections (STIs) [6, 7]. In 2011, youth aged 13-24 accounted for approximately $21 \%$ of all new HIV infections in the USA and young adults aged 20-24 experienced the highest rates of HIV, chlamydia, gonorrhea, and syphilis [6, 7]. That same year, MSM accounted for $79 \%$ of HIV diagnoses among all males aged 13 years and older, and $62 \%$ of diagnoses among all persons diagnosed with HIV [7]. Research on online social networking in relation to sexual behaviors has focused on a number of specific outcomes, such as individual representations of sexuality via online platforms $[8,9]$, transmission of social norms that are associated with sexual behaviors $[10 \cdot 11]$, and the facilitation of sexual contact with partners met online $[12 \cdot, 13]$. Because research on the relationship between online social networking and sexual behaviors is an emerging area of interest for clinicians and researchers, we will include studies in this review with a variety of sexual behavior outcomes; however, our primary interest will be studies that examined a behavioral risk outcome that can be directly tied with STI acquisition or transmission (e.g., condomless sex).

Many different types of interactions between individuals that occur via internet platforms may be thought of as social networking. However, in keeping with recent trends in the extant literature, we use Boyd and Ellison's criteria [14] for SNS to narrow the focus of possible online interactions between individuals. For purposes of this review, we will define SNS as platforms that allow users to do the following: (1) construct profiles within a bounded system; (2) articulate a list of other users with whom they share a tie; and (3) view and traverse their list of connections and connections made by others within the system. As such, our review includes mainstream SNS (e.g., Facebook, Twitter), online dating and "partner seeking" sites (e.g., Match.com, Manhunt.com), and geosocial networking applications ("apps") (e.g., Grindr, Tinder). In the interest of parsimony, we will not cover communication via text message (e.g., "sexting"), communication via chatroom or online message boards or other types of technology-based communication. Although some studies have linked these latter types of communication to sexual risk behaviors, they generally do not take place via SNS platforms as defined above. In studies that include SNS use along with other types of online social networking, we have tried to be as specific as possible in the presentation of findings and their implications for clinicians and researchers.

\section{Methods}

Social networking technologies evolve rapidly, presenting challenges to researchers and consumers with regard to use and evaluation of the most current and most popular platforms; and further complicating systematic and rigorous evaluation of the behaviors associated with these technologies. For this review, studies were identified by searching for peerreviewed publications in Google Scholar and PubMed, and through consultations with experts in the field. We used the following search terms: "online social networking," AND "sexual risk behavior(s)," "sexual risk behaviour(s)," "sexually transmitted infection(s)," and "sexual risk." The Google Scholar search resulted in a total of 214 unique citations. Two independent reviewers (HDP and $\mathrm{KH}$ ) screened all citations based on the title and abstract to assess for applicability; disagreements between reviewers were resolved by third party adjudication (IWH). After reviewing the full text article, 37 US-based studies that dealt specifically with online social networking and sexual risk and protective behaviors were identified (Fig 1). Those highlighted in the current paper are those we deemed most important to the field because they extend prior work and offer new information on this

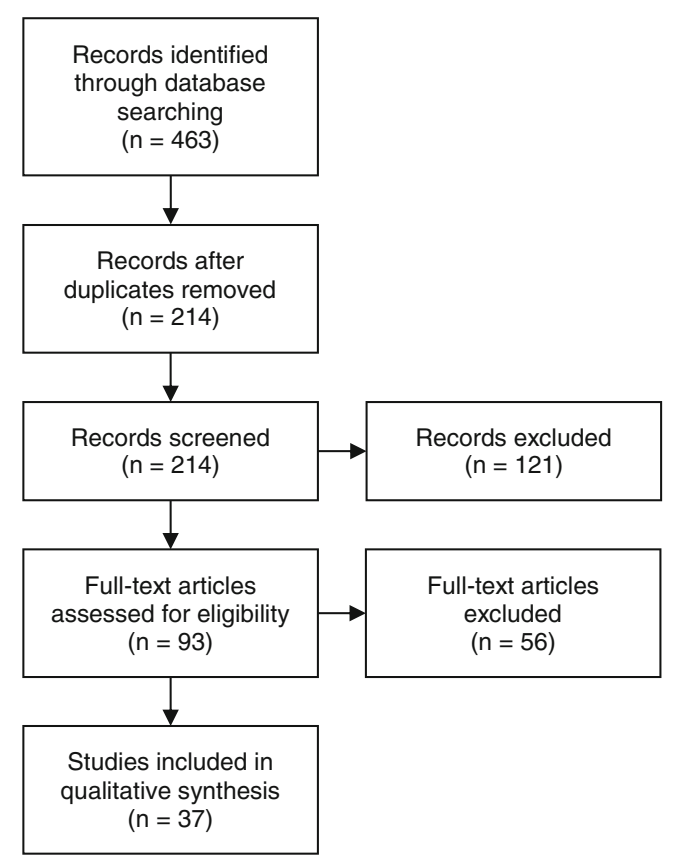

Fig. 1 Systematic literature review for online sexual networking and sexual risk and protective behaviors 
relationship. In this review, we first provide an overview of important findings on the association between online social networking and sexual risk and protective behaviors. Next, we offer practical advice for clinicians and researchers who may be interested in discussing online social networking with patients, exploring health outcomes associated with online social networking, and incorporating these platforms into clinical practice or future research studies.

\section{Results}

\section{Mainstream Social Networking Sites (SNS)}

Facebook is by far the most popular SNS in the USA. Roughly $71 \%$ of US adults online use Facebook and approximately $63 \%$ of Facebook users visit the site daily (with $40 \%$ visiting Facebook multiple times per day) [3]. However, Americans are increasingly diversifying their SNS usage according to various elements of their personality, preferences, and behaviors. For people who use a single SNS, Facebook is the platform of choice; however, one half of adults online use multiple SNS, including Instagram, Pinterest, and Twitter [3]. Both Instagram and Twitter have experienced marked increases in online usage since 2012 , with nearly $60 \%$ of Instagram users checking the site daily, and nearly $50 \%$ of Twitter users doing the same [3]. Additionally, both Instagram and Twitter usage has increased among African-American internet users and those 18-29 years of age since 2012, from $13 \%$ to $18 \%$ [3]. These data suggest both the popularity and widespread integration of SNS in the daily lives of Americans. Indeed, some have argued that these virtual environments have become an extension of, and in some cases replaced, in-person social connections [15].

To date, Facebook, Myspace and Twitter have been the primary SNS that have been examined in relation to sexual risk behaviors. Myspace in particular has been the subject of early studies on adolescent sexual behavior as reviewed in a report on the influence of new media and adolescent sexual health conducted by RAND Health. A study by Hindjua and Patchin [16] examined a random sample of nearly 1,500 public MySpace profiles of 16-17 year old users and found that small percentages of profiles contained pictures with some nudity ( $5 \%$ of users themselves; $15 \%$ of friends or family). Moreno and colleagues [17] performed a content analysis of 500 publicly available profiles of youth 18 years of age and found that $24 \%$ of profiles contained "personal sexual preferences, self-disclosures of sexual experiences, (and) pictures of profile owner in undergarments." (pp. 2930 ). Young women were marginally more likely to post sexual content than their male counterparts. In addition, espousing a sexual identity other than heterosexual was associated with more references to sexual behavior. Williams and Merton [18] coded 100 MySpace profiles for sexual content and found that $16 \%$ of profiles contained references to sexual activity. In a more recent study of another teen SNS website (MyLol.net), Pujazon-Zazik, et al. [9], found that $16 \%$ of over 750 publicly viewable profiles of adolescents ages 14-18 contained references to sexual behavior. While none of these studies linked SNS use with sexual risk behaviors, they suggest that for some young people, SNS are sexualized environments.

More recently, literature on SNS in relation to sexual risk behavior among youth has expanded beyond surveying profiles for sexualized context to examining SNS use patterns and content of SNS-based communication that may lead to sexual behavior. Whitely, et al. [19•], examined technology use among 1,500 African American adolescents aged 13-17 years old, recruited via community-based sampling in the northeastern and southeastern USA, and found that $60 \%$ used SNS most days (96\% used MySpace, $27 \%$ Facebook), and that internet use (including SNS use) was associated with increased sexual sensation seeking, but not increased peer sexual risk norms. A study of homeless youth (ages 13-24), recruited from social service agencies in Los Angeles, demonstrated that type of online communication is an important predictor for both STI risk and protective behaviors. While SNS use (78 \% used MySpace, $30 \%$ Facebook, $10 \%$ Twitter) was associated with having been tested for STIs, discussing safer sex via SNS was associated with both increased HIV knowledge and having met more sexual partners online; and having online sex partners and talking to friends via SNS about drugs and partying was associated with increased exchange sex [20]. Young and Jordan [11] found that college students assigned to view sexually suggestive Facebook photos reported the perception that larger percentages of their peers had unprotected sexual intercourse and sex with strangers and were more likely to report willingness to engage in these behaviors themselves, compared to those who did not view sexually suggestive photos. Together, these studies reflect the importance of considering communication content when evaluating online interactions - the topics young people discuss with their friends, and the photos posted by their friends, may play an important role in shaping offline behaviors that promote or deter sexual risk behaviors.

More traditional sexual risk behavior outcomes (e.g., number of sexual partners, unprotected sexual intercourse) have also been estimated in relation to SNS use among young people and some studies have found evidence for a relationship between increased sexual risk behaviors and SNS use. For example, Buhi, et al. [21], surveyed adolescents (aged 1319) from a publicly funded teen clinic in Florida at which $80 \%$ of those with online partners met them through SNS (MySpace, Facebook, and various others), and found that meeting a sexual partner online was associated with vaginal sex before age 14, greater numbers of vaginal sex partners, alcohol use during or immediately before sex, and same-sex 
sexual activity among adolescents. Black, Schmiege and Bull [10॰] determined associations between sexual risk (defined as condomless sex, multiple partners, concurrent partners, sexual pressure, alcohol and drug use during sex) and higher perceptions of these behaviors by peers in Facebook networks of 1625 year olds. These findings suggest that actual online behavior and perceived behavior of peers in SNS-based networks may play an important role in determining sexual risk behaviors of young people. Findings of these cross-sectional studies should be fortified by more robust study designs that can further elucidate temporal sequencing between individual risk behavior and online social networking.

Twitter has also been used to document sexual risk behaviors and disseminate sexual health information. Young, Rivers and Lewis [22•] analyzed 553,186,061 tweets and determined a positive association between sexual tweeting and HIV prevalence by US county, suggesting that aggregate social networking data may serve as a method for evaluation and detection of HIV risk behaviors across large geographic regions. This study demonstrates the utility of SNS data in epidemiological studies, but does not offer insights into individual Twitter use and sexual risk behaviors. The Get Yourself Tested (GYT) campaign, a collaboration between MTV, Kaiser Family Foundation and Planned Parenthood, used Twitter (along with Facebook and other SNS) to augment "on the ground" events aimed at increasing STD testing among youth, and documented increased chlamydia testing at Planned Parenthood affiliates following implementation; although it is difficult to determine whether this was a function of engagement via SNS [23]. Twitter, like Instagram, Pinterest, Google+, FourSquare, and other increasingly popular SNS, remains largely unexplored in the academic literature. In the coming years, it is certain that new SNS will emerge and existing sites may offer varied functionality that may promote (or deter) sexual risk behaviors of users. Researchers interested in the association between mainstream SNS and sexual risk behavior should consider addressing gaps in the literature with an eye toward findings that will generalize across sites. Greater attention to peer influence processes via SNS (i.e., sexual health communication, social norms creation, bullying via SNS), are needed.

\section{Internet Dating, "Sex Seeking” and "Hook-up" Sites}

While the literature on mainstream SNS use and sexual risk behaviors emerges, research on online partner-seeking via dating, "sex seeking" and "hook up" sites is more established. This is especially true for research on the sexual risk behaviors of MSM who have used the internet for partner seeking since the early 1990s [24••]. SNS designed for partner-seeking among MSM have grown popular because they give users the ability to: (a) conveniently and quickly locate sexual partners [25]; (b) sort opportunities for sex by partner characteristics and preferences [26]; (c) control information about sexual preferences and behaviors [27]; and (d) engage with a community of similar individuals that can provide social support free from stigmatized reactions from friends and family [27-29]. A study of 2,505 MSM from the UK demonstrated that from 1993 to 2002, the percentage of MSM who met their first male sexual partner through the internet increased from $3 \%$ to $61 \%$ [30]; at the same time percentages of MSM who met their partners in gay venues such as bars and clubs declined from $34 \%$ to $17 \%$. SNS designed for MSM have become a prime venue for socialization and sexual partner seeking in this population.

Despite a significant body of research, the relationship between online social networking and sexual risk among MSM remains unclear. This may be due, in large part, to variation across studies in sampling and lack of specificity about sites in which online partner seeking occurs. While several studies have demonstrated a positive association between online partner-seeking and greater sexual risk behaviors among MSM [31-33], others have found no relationship between these variables $[34,35]$. Specific work on the relationship between seeking romantic and/or sexual partners online and risk behaviors has provided some additional insights. Mustanski, et al. [36], found that meeting a sex partner online was not associated with increased unprotected anal intercourse (UAI) among young MSM (YMSM) recruited using snowball sampling in a large Midwestern city. Similar results were found in a national sample of internet-using MSM [37], and in a 12-city study of MSM recruited in person [38 ${ }^{\bullet}$. However, a subgroup analysis in the latter study revealed that rural MSM who had used the internet to find their last sex partner were nearly twice as likely to report UAI, than their urban counterparts. One of the limitations of these studies is that they do not take into account the variety and types of online venues in which MSM meet their partners, thereby grouping SNS with other types of platforms, such as message boards and chatrooms.

Two recent studies examining online partner seeking and sexual risk behavior among MSM further elucidate the relationship between online partner seeking and sexual risk behaviors. Bauermeister, et al. [39], grouped YMSM who used the internet to seek partners by number of romantic versus casual partners sought online (high romantic/high casual, low romantic/high casual, etc.), and found that YMSM in the high romantic/high casual group were more likely to engage in UAI than any other group. Downing [40] demonstrated that among MSM who use the internet for partner-seeking, highfrequency internet users attended sex venues (e.g., gyms, public bathrooms, sex clubs) more often than low-frequency users, and were more likely to engage in UAI across venues. These findings suggest that gay, bisexual and other MSM who have a history of, or higher propensity for, engaging in sexual risk behaviors may find SNS an efficient way to meet partners 
with whom they can engage in these activities. This research underscores the need to further evaluate whether associations between SNS and sexual risk behaviors are dictated by selection (e.g., whether people with a diathesis for behavior find a magnifying effect due to pervasiveness, ease and anonymity of use), or influence (e.g., whether characteristics of certain SNS actually promote risk behaviors).

\section{Geosocial Networking Applications "Apps"}

Geosocial networking apps have emerged as new platforms by which users are chatting, dating, and meeting for sex. These applications are unique, compared to traditional online dating sites or mainstream computer-based SNS, in that they rely on the geolocation feature of smartphones to enable users to connect with each other, based on geographic proximity. Apps like Grindr and Tinder, for example, have become especially popular because they let users know whether another user is within 500 feet or miles away. Based on our review, the only research documenting the association between these mobile SNS and sexual risk behaviors have been conducted among MSM. This work demonstrates that these apps are used for a variety of reasons, including friendship, dating, sexual encounters, "killing time," and connecting to the larger gay community [12•, 41]. A study by Holloway, et al. [42], compared Grindr use to both Facebook and dating site use among YMSM (aged 18-24) and found numerous differences in motivations for using these varied social networking platforms. For example, participants were more likely to use Facebook to connect with face-to-face friends but more likely to use Grindr to make new friends. Participants were also more likely to use gay dating sites to meet people with whom to hook-up compared to Grindr.

Results on the association between sexual risk behavior and app use are mixed, with some studies indicating greater lifetime and recent sex partners among those recruited via app versus others [43], and app-users versus non-app users [13]. Landovitz, et al. [12•], found that in a sample of 375 MSM, $46 \%$ of app users engaged in recent ( 3 month) UAI with any partner (met via app or elsewhere), and $39 \%$ reported unprotected receptive anal intercourse (URAI). Grosskopf, LeVasseur, \& Glaser [44], and Lehmiller \& Ioerger [13], found no significant differences in sexual risk behavior between MSM who met partners via apps, compared to MSM who met partners on other internet platforms. Rice, et al. [41], found that app users were more likely to use condoms with app-met partners, than with partners met elsewhere. However, those who reported UAI with their last app-met partner had a greater number of lifetime and recent sex partners, compared to those who did not report UAI with an app-met partner. Data published on the same sample indicated that UAI among appmet partners was associated with longer duration of app use, more sexually explicit profile photos, and more past month app-met partners [45].

Among MSM using apps to meet sexual partners, the popularity and accessibility of these apps may foster opportunities to meet many more sexual partners than traditional internet platforms or in-person settings, such as bars and clubs. As geosocial networking apps increase in popularity, more research is needed on the usage patterns and content of communication among diverse MSM. All of the studies to date have been conducted among urban samples of app-users. However, the opportunities for social connection and sexual partnering may differ in rural communities, where MSM may experience greater difficulties meeting other MSM than in large urban settings with traditional gay neighborhoods. The existing literature on geosocial networking apps also lacks specificity regarding usage patterns across racial/ethnic groups. The largest racial/ethnic groups represented in studies on app use have been white MSM (with no analyses stratified by race/ethnicity), leaving much unknown about usage patterns and associated risks that are specific to racial/ethnic minority men, a group at increased risk for HIV infection. Others have noted differences in sexual risk behaviors by MSM recruited via diverse SNS [46], but few have extended their analyses to include popular apps. Finally, geosocial networking apps that cater to heterosexual (as well as MSM) communities, such as Tinder, are unexplored in the literature to date; examination of these SNS platforms and their association with risk and protective behaviors is warranted.

\section{Recommendations for Clinicians and Researchers}

SNS present opportunities for both risk and protective behaviors related to sex. The ability to connect with strangers for sexual encounters may lead to increases in number of sexual partners, but may also promote conversations related to safer sex and expectations for reduced risk behavior that can be difficult to negotiate in person [47]. SNS also offer a prime opportunity for public health researchers and clinicians, who are interested in promoting safer sex, to reach a large number of individuals, and tap into networks where health promotion messaging may diffuse rapidly. In 2011, Ralph, et al. [48], examined the acceptability of using MySpace to reach youth (ages 14 to 19) for sexual health services, and found that $41 \%$ were willing to accept a friend request from a sexual health clinic (37\% were uncertain), and $50 \%$ were (very or somewhat) interested in receiving information via MySpace or another SNS. The literature on app use among MSM also highlights opportunities for HIV testing [49], and the feasibility and acceptability of app-based recruitment and intervention $[42,43,50]$. For example, Holloway et al., found that $70 \%$ of YMSM were willing to receive an HIV prevention intervention via smartphone app [42]. Hightow-Weidman and colleagues [51•] are already using SNS to notify partners of 
patients who tested positive for HIV and syphilis infection in North Carolina, and the GYT campaign (described above) was successful in reaching thousands of youth through Facebook, Twitter, and other SNS [23], demonstrating that interventions for prevention and treatment are feasible and acceptable.

In addition to evaluation of the GYT campaign [23], additional studies have been published using Facebook to deliver sexual health promotion and HIV risk behavior prevention messaging to young people [52.], and African American and Latino MSM [53]. Bull, et al. [52•], evaluated an SNS-based intervention for youth (ages 18 to 24) involving weekly postings on a Facebook page that included communication regarding sexual history, expectations of healthy relationships, skills building for condom use negation and condom use, and how to access STI testing. Compared to a control group, those who received the intervention had greater condom use over a 2month (but not a 6-month) period. Young, et al. [53], randomly assigned African American and Latino MSM to a private Facebook group where they received either HIV-prevention messaging (intervention), or general health messaging (control), from trained online peer opinion leaders. Network ties within groups increased over time, and were associated with more requests for home-based HIV test kits and decreased sexual risk behavior among those in the intervention [54•]. These studies demonstrate the efficacy of SNS-based interventions to promote safer sexual behaviors among young people and MSM, especially over short periods of time. Further refinement and effectiveness testing for these, and similar SNS-based interventions, as well as continued work in theory development to link SNS use with behavior change, and integration of multiple data sources for evaluation of SNSbased interventions [55], are necessary for promotion of safer sexual behaviors among SNS-using populations.

Clinicians who want to incorporate SNS-based interventions in their practice may find the first-generation SNS-based studies highlighted above promising; however, a need for more robust research to understand the impact of SNS on sexual risk and protective behaviors will greatly benefit the tailoring and implementation of future efforts [56]. In addition, with opportunities to reach large numbers of patients via SNS come challenges about how to deliver sexual health information in a manner that is respectful, and takes into consideration ethical challenges related to privacy and confidentiality. Recent work lays out ethical challenges for HIV research, including ways in which to contact users that protects unwanted disclosure of personal information [26, 57••]. This work is highly relevant for clinical providers, who may be unaware of the ways in which background user information collected via SNS can be accessible and/or sold to third-party advertisers. Any clinician wishing to advertise, approach clients, or maintain ongoing online communication via SNS, should be aware of the user agreements and privacy polices of individual SNS, and should remain abreast of updates to those policies, and the implications for clients and patients. Clinicians should also clearly communicate the potential limitations of using SNS technologies to their clients and patients, prior to using SNS in sexual risk reduction interventions, as well as follow the laws and regulations of the Health Insurance Portability and Accountability Act (HIPAA), particularly when contacting individuals. Advertising or promoting messages or services via SNS, but providing means of acquiring additional information outside of SNS, seems to be the current best-practice for maintaining confidentiality and privacy; such efforts might include providing telephone, email, or program/ project-specific URL links, as part of SNS-based marketing/ promotion.

Since MSM are prime targets for STI/HIV prevention via SNS, based on their disproportionate rates of disease burden and widespread SNS use, clinicians working with this population should be especially sensitive to the unique needs of these men. Rosenberger and colleagues (2011) remind us of the importance of "sex positive" messaging on SNS targeting MSM. Sullivan, Grey, and Rosser [58] highlight the need for tailored, technology-based HIV-prevention for MSM that has varied functionality, and combines both technology-delivered components with in-person components, based on the needs of the target population. This is an important point to remember for any clinician who wishes to develop SNS-based sexual health interventions: technology should not necessarily replace in-person services. For each client or patient population a careful initial assessment of that populations' technology use patterns and comfort should be assessed, and clinicians should reassess client preferences and use patterns throughout the intervention for potential changes. Finally, when working with youth and MSM, clinicians should consider initiating conversations about the role of SNS use in sexual partner seeking. Dialogues with patients about their use of SNS may help identify health risks and potential SNS and non-SNS opportunities for health promotion and STI/HIV prevention among individual clients and patients.

\section{Conclusions}

This review of recent literature regarding the relationship between online social networking and sexual risk and protective behaviors provides a background for clinicians and researchers who are interested in learning more about these technologies, their benefits and drawbacks, and how SNS might be harnessed for advances in health behavior research and clinical care. While SNS have the potential to be powerful tools to promote sexual health, sex positivity, disease prevention, and linkage to care and treatment, they also have the capacity to become risky environments that can compromise interpersonal skills, promote risky norms around sexual 
behaviors, and foster disease spread. How to harness the power of SNS for population health and individual health promotion will require new strategies for rapidly evaluating fast-moving technologies systematically and using harmonized outcomes and assessments which will require unparalleled cooperation between funding agencies, communities, researchers, and technology experts.

Acknowledgments This research was supported by the Center for HIV Identification, Prevention, and Treatment Services (CHIPTS) NIMH grant P30MH058107; the UCLA AIDS Institute and the UCLA Center for AIDS Research (CFAR) NIH grant P30AI028697; and the National Center for Advancing Translational Sciences through UCLA CSTI grant UL1TR000124. The content is solely the responsibility of the authors and does not necessarily represent the views of the official views of the NIH.

Dr. Landovitz acknowledges support from the National Institute of Drug Abuse (K23DA026308) of the National Institutes of Health.

The authors wish to acknowledge the assistance of Joanna Barreras, Joshua Rusow and Ashley Schuyler in the preparation of this manuscript.

\section{Compliance with Ethics Guidelines}

Conflict of Interest Dr. Holloway reports grants from National Institute on Drug Abuse and grants from National Institute of Mental Health, during the conduct of the study.

Dr. Landovitz reports grants from the National Institute on Drug Abuse, the National Institute of Mental Health, and the California HIV/ AIDS Research Program during the conduct of the study.

Homero E. del Pino, Craig Pulsipher, Shannon Dunlap and Keith Hermanstyne declare that they have no conflict of interest.

Human and Animal Rights and Informed Consent This article does not contain any studies with human or animal subjects performed by any of the authors.

\section{References}

Papers of particular interest, published recently, have been highlighted as:

- Of importance

•. Of major importance

1. Brenner J, Smith A. $72 \%$ of online adults are social networking site users. Washington, DC: Pew Research Center; 2013.

2. Pew Research Global Attitudes Project. Emerging nations embrace internet, mobile technology. Pew Research Center. 2014. http:// www.pewglobal.org/2014/02/13/emerging-nations-embraceinternet-mobile-technology/. Accessed May 102014.

3. Duggan M, Smith A. Social media update 2013. Washington, DC: Pew Research Center; 2013.

4. Pew Research Internet Project. Social networking fact sheet. Pew Research Center. 2013. http://www.pewinternet.org/fact-sheets/ social-networking-fact-sheet/. Accessed May 72014.

5. Pew Research Internet Project. Social media use over time. Pew Research Center. 2014. http://www.pewinternet.org/data-trend/ teens/social-media/. Accessed May 102014.

6. Centers for Disease Control and Prevention. Sexually transmitted disease surveillance 2011. Atlanta: US Department of Health and Human Services; 2012.
7. Centers for Disease Control and Prevention. HIV surveillance report, 2011. Atlanta: US Department of Health and Human Services; 2013.

8. Noll JG, Shenk CE, Barnes JE, Haralson KJ. Association of maltreatment with high-risk internet behaviors and offline encounters. Pediatrics. 2013;131(2):e510-7.

9. Pujazon-Zazik MA, Manasse SM, Orrell-Valente JK. Adolescents' self-presentation on a teen dating web site: a risk-content analysis. J Adolesc Health. 2012;50(5):517-20.

10. Black SR, Schmiege S, Bull S. Actual versus perceived peer sexual risk behavior in online youth social networks. Transl Behav Med. 2013;3(3):312-9. Demonstrates relationship between perceptions of online peers' behaviors and reporting of risk behaviors among a large sample of 16-25 years old Facebook users.

11. Young SD, Jordan AH. The influence of social networking photos on social norms and sexual health behaviors. Cyberpsychol Behav Soc Netw. 2013;16(4):243-7.

12. Landovitz RJ, Tseng C-H, Weissman M, Haymer M, Mendenhall B, Rogers K, et al. Epidemiology, sexual risk behavior, and HIV prevention practices of men who have sex with men using GRINDR in Los Angeles, California. J Urban Health. 2013;90(4):729-39. Among the first studies to examine geosocial networking app use among MSM and highlights the acceptability of combined behavioral and biological HIV prevention interventions among app-using MSM.

13. Lehmiller JJ, Ioerger M. Social networking smartphone applications and sexual health outcomes among men who have sex with men. PLoS One. 2014;9(1):e86603.

14. Boyd DM, Ellison NB. Social network sites: definition, history, and scholarship. J Comput Mediat Commun. 2007;13(1):210-30.

15. Zhang Y, Leung L. A review of social networking service (SNS) research in communication journals from 2006 to 2011. New Media Soc. 2014. doi: $10.1177 / 1461444813520477$.

16. Hinduja S, Patchin JW. Personal information of adolescents on the Internet: a quantitative content analysis of MySpace. J Adolesc. 2008;31(1):125-46.

17. Moreno MA, Parks MR, Zimmerman FJ, Brito TE, Christakis DA. Display of health risk behaviors on MySpace by adolescents: prevalence and associations. Arch Pediatr Adolesc Med. 2009;163(1):27-34.

18. Williams AL, Merten MJ. A review of online social networking profiles by adolescents: implications for future research and intervention. Adolescence. 2008;43(170):253-74.

19. Whiteley LB, Brown LK, Swenson RR, Romer D, DiClemente RJ, Salazar LF, et al. African American adolescents and new media: associations with HIV/STI risk behavior and psychosocial variables. Ethn Dis. 2011;21(2):216-22. Links greater internet (including SNS) use with a history of oral/vaginal/anal sex among a large sample of African American adolescents.

20. Young SD, Rice E. Online social networking technologies, HIV knowledge, and sexual risk and testing behaviors among homeless youth. AIDS Behav. 2011;15(2):253-60.

21. Buhi ER, Klinkenberger N, McFarlane M, Kachur R, Daley EM, Baldwin J, et al. Evaluating the internet as a sexually transmitted disease risk environment for teens: findings from the communication, health, and teens study. Sex Transm Dis. 2013;40(7):528-33.

22. Young SD, Rivers C, Lewis B. Methods of using real-time social media technologies for detection and remote monitoring of HIV outcomes. Prev Med. 2014. doi:10.1016/j.ypmed.2014.01.024. Uses Twitter feeds and existing HIV reporting to document a relationship between sexual tweets and HIV prevalence. Example of how big data can be harnessed for evaluation and detection of HIV risk behaviors in real-time.

23. Friedman AL, Brookmeyer KA, Kachur RE, Ford J, Hogben M, Habel MA, et al. An assessment of the GYT: get yourself tested 
campaign: an integrated approach to sexually transmitted disease prevention communication. Sex Transm Dis. 2014;41(3):151-7.

24.• Grov C, Breslow AS, Newcomb ME, Rosenberger JG, Bauermeister JA. Gay and bisexual men's use of the internet: research from the 1990s through 2013. Am J Mens Health. 2014;51(4):390-409. Provides a comprehensive history of how MSM have used the internet (including SNS) to meet sexual partners and offers an excellent review of work linking online partner seeking to sexual risk behaviors among MSM.

25. Rosser BS, Oakes JM, Horvath KJ, Konstan JA, Danilenko GP, Peterson JL. HIV sexual risk behavior by men who use the internet to seek sex with men: results of the Men's INTernet Sex Study-II (MINTS-II). AIDS Behav. 2009;13(3):488-98.

26. Bull SS, Breslin LT, Wright EE, Black SR, Levine D, Santelli JS. Case study: an ethics case study of HIV prevention research on facebook: the Just/Us study. J Pediatr Psychol. 2011;36(10):1082-92.

27. Brown G, Maycock B, Burns S. Your picture is your bait: use and meaning of cyberspace among gay men. J Sex Res. 2005;42(1):63-73.

28. Benotsch EG, Kalichman S, Cage M. Men who have met sex partners via the internet: prevalence, predictors, and implications for HIV prevention. Arch Sex Behav. 2002;31(2):177-83.

29. Robinson BA, Moskowitz DA. The eroticism of Internet cruising as a self-contained behaviour: a multivariate analysis of men seeking men demographics and getting off online. Cult Health Sex. 2013;15(5):555-69.

30. Bolding G, Davis M, Hart G, Sherr L, Elford J. Where young MSM meet their first sexual partner: the role of the internet. AIDS Behav. 2007;11(4):522-6.

31. Berry M, Raymond HF, Kellogg T, McFarland W. The internet, HIV serosorting and transmission risk among men who have sex with men, San Francisco. AIDS. 2008;22(6):787-9.

32. Garofalo R, Herrick A, Mustanski BS, Donenberg GR. Tip of the iceberg: young men who have sex with men, the internet, and HIV risk. Am J Public Health. 2007;97(6):1113.

33. Horvath KJ, Bowen AM, Williams ML. Virtual and physical venues as contexts for HIV risk among rural men who have sex with men. Health Psychol. 2006;25(2):237.

34. Chiasson MA, Hirshfield S, Remien RH, Humberstone M, Wong T, Wolitski RJ. A comparison of on-line and off-line sexual risk in men who have sex with men: an event-based on-line survey. J Acquir Immune Defic Syndr. 2007;44(2):235-43.

35. Jenness SM, Neaigus A, Hagan H, Wendel T, Gelpi-Acosta C, Murrill CS. Reconsidering the internet as an HIV/STD risk for men who have sex with men. AIDS Behav. 2010;14(6):1353-61.

36. Mustanski B, Lyons T, Garcia SC. Internet use and sexual health of young men who have sex with men: a mixed-methods study. Arch Sex Behav. 2011;40(2):289-300.

37. Grov C, Hirshfield S, Remien RH, Humberstone M, Chiasson MA. Exploring the venue's role in risky sexual behavior among gay and bisexual men: an event-level analysis from a national online survey in the US. Arch Sex Behav. 2013;42(2):291-302.

38. Kakietek J, Sullivan P, Heffelfinger J. You've got male: internet use, rural residence, and risky sex in men who have sex with men recruited in 12 U.S. cities. AIDS Educ Prev. 2011;23(2):118-27. Offers evidence that rural MSM who used the internet to find their last sex partners may be at greater risk of STI/HIV infection than their urban counterparts in a 12-city study of MSM.

39. Bauermeister JA, Leslie-Santana M, Johns MM, Pingel E, Eisenberg A. Mr. Right and Mr. Right Now: romantic and casual partner-seeking online among young men who have sex with men. AIDS Behav. 2011;15(2):261-72.

40. Downing MJ. Using the internet in pursuit of public sexual encounters: is frequency of use associated with risk behavior among MSM? Am J Mens Health. 2012;6(1):18-27.

41. Rice E, Holloway I, Winetrobe H, Rhoades H, Barman-Adhikari A, Gibbs J, et al. Sex risk among young men who have sex with men who use Grindr, a smartphone geosocial networking application. J AIDS Clin Res. 2012;S4:005. doi:10.4172/2155-6113.S4-005.

42. Holloway IW, Rice E, Gibbs J, Winetrobe H, Dunlap S, Rhoades H. Acceptability of smartphone application-based HIV prevention among young men who have sex with men. AIDS Behav. 2014;18(2):285-96.

43. Burrell ER, Pines HA, Robbie E, Coleman L, Murphy RD, Hess $\mathrm{KL}$, et al. Use of the location-based social networking application GRINDR as a recruitment tool in rectal microbicide development research. AIDS Behav. 2012;16(7):1816-20.

44. Grosskopf NA, LeVasseur MT, Glaser DB. Use of the internet and mobile-based "apps" for sex-seeking among men who have sex with men in New York City. Am J Mens Health. 2014: 1557988314527311.

45. Winetrobe H, Rice E, Bauermeister J, Petering R, Holloway IW. Associations of unprotected anal intercourse with Grindr-met partners among Grindr-using young men who have sex with men in Los Angeles. AIDS Care. 2014:1-6. doi:10.1080/09540121.2014. 911811.

46. Vial AC, Starks TJ, Parsons JT. Finding and recruiting the highest risk HIV-negative men who have sex with men. AIDS Educ Prev. 2014;26(1):56-67.

47. Veinot TC, Campbell TR, Kruger D, Grodzinski A, Franzen S, editors. Drama and danger: the opportunities and challenges of promoting youth sexual health through online social networks. AMIA Annual Symposium Proceedings. Washington, DC: American Medical Informatics Association; 2011.

48. Ralph LJ, Berglas NF, Schwartz SL, Brindis CD. Finding teens in their space: using social networking sites to connect youth to sexual health services. Sex Res Soc Policy. 2011;8(1):38-49.

49. Rendina HJ, Jimenez RH, Grov C, Ventuneac A, Parsons JT. Patterns of lifetime and recent HIV testing among men who have sex with men in New York City who use Grindr. AIDS Behav. 2014;18(1):41-9.

50. Phillips II G, Magnus M, Kuo I, Rawls A, Peterson J, Jia Y, et al. Use of geosocial networking (GSN) mobile phone applications to find men for sex by men who have sex with men (MSM) in Washington, DC. AIDS Behav. 2014:1-8. doi:10.1007/s10461014-0760-9.

51. Hightow-Weidman L, Beagle S, Pike E, Kuruc J, Leone P, Mobley V, et al. "No one's at home and they won't pick up the phone": using the internet and text messaging to enhance partner services in North Carolina. Sex Transm Dis. 2014;41(2):143-8. Demonstrates the feasibility utility for partner's notification of HIV and syphilis infection via SNS.

52. Bull SS, Levine DK, Black SR, Schmiege SJ, Santelli J. Social media-delivered sexual health intervention: a cluster randomized controlled trial. Am J Prev Med. 2012;43(5):467-74. Uses a rigorous study design to evaluate the efficacy of a Facebook intervention to promote sexual health among youth and finds promising shortterm outcomes related to condom use behavior.

53. Young SD, Szekeres G, Coates T. Sexual risk and HIV prevention behaviours among African-American and Latino MSM social networking users. Int J STD AIDS. 2013;24(8): 643-9.

54. Young SD, Holloway IW, Jaganath D, Rice E, Westmoreland D, Coates T. Online social network changes in an HIV prevention RCT for African American and Latino men who have sex with men. Am J Public Health. 2014. Demonstrates that structural network mechanisms are associated with HIV testing behaviors among racial ethnic minority MSM enrolled in a Facebook-delivered RCT.

55. Bull SS. Sexually transmitted disease prevention campaigns in the 21st century: new frontiers in social media. Sex Transm Dis. 2014;41(3):158-60.

56. Allison S, Bauermeister JA, Bull S, Lightfoot M, Mustanski B, Shegog R, et al. The intersection of youth, technology, and new 
media with sexual health: moving the research agenda forward. $\mathrm{J}$ Adolesc Health. 2012;51(3):207-12.

57.• Curtis BL. Social networking and online recruiting for HIV research: ethical challenges. J Empir Res Hum Res Ethics. 2014;9(1):58-70. Outlines key ethnical considerations for researchers (that are also highly relevant for clinicians) regarding technology-based communication via SNS.

58. Sullivan PS, Grey JA, Rosser BRS. Emerging technologies for HIV prevention for MSM: what we have learned, and ways forward. J Acquir Immune Defic Syndr. 2013;63:S102-7. 